Cuestiones de sociología

ISSN: 2346-8904

publicaciones@fahce.unlp.edu.ar

Universidad Nacional de La Plata

Argentina

\title{
"Terapias del aquí y ahora porque lo que te pasa te pasa hoy": brevedad y eficacia como modelo de abordaje y resolución en los tratamientos cognitivo-conductuales
}

\author{
Del Monaco, Romina \\ "Terapias del aquí y ahora porque lo que te pasa te pasa hoy": brevedad y eficacia como modelo de \\ abordaje y resolución en los tratamientos cognitivo-conductuales \\ Cuestiones de sociología, núm. 22, 2020 \\ Universidad Nacional de La Plata, Argentina \\ DOI: https://doi.org/10.24215/23468904e090
}

Atribución no comercial compartir igual (CC BY-NC-SA) 4.0 
Artículos

\section{"Terapias del aquí y ahora porque lo que te pasa te pasa hoy": brevedad y eficacia como modelo de abordaje y resolución en los tratamientos cognitivo- conductuales}

"Here and now therapies because what happens to you it happens now": brevity and efficacy as a model of approach
and resolution in cognitive behavioral treatments

Romina Del Monaco

DOI: https://doi.org/10.24215/23468904e090

Instituto de Investigaciones Gino Germani-Facultad

de Ciencias Sociales (Universidad de Buenos Aires-

CONICET), Argentina

rominadelmonaco@gmail.com

\section{Resumen:}

Partiendo de una investigación sobre terapias cognitivo-conductuales en la Ciudad Autónoma de Buenos Aires, este artículo tiene por objetivo explorar y analizar en las narrativas de los/as profesionales entrevistados/as qué lugar tiene la brevedad como un aspecto que atraviesa y modela el proceso terapéutico. Desde el abordaje cognitivo-conductual se resalta el hecho de ser una psicología que realiza investigaciones basadas en la evidencia y esta cuestión permite estipular los plazos de los tratamientos. Más aún, se señala que es posible "resolver problemas" en determinado tiempo y que, si bien pueden sufrir modificaciones y prolongarse o acortarse, hay regularidades que se mantienen y, de algún modo, preexisten a las experiencias de quienes padecen. A partir de lo señalado, interesa enmarcar y analizar la relación entre brevedad y eficacia teniendo en cuenta una serie de transformaciones sociales y económicas dentro de las cuales estas psicoterapias surgen y adquieren mayor protagonismo. Se trata de contextualizar su desarrollo considerando, en las sociedades actuales, las exigencias a los sujetos para cumplir en su cotidianeidad simultáneamente con distintas tareas (personales, vinculares, laborales, etc.) lo más rápido posible. Y, a su vez, tener en cuenta que la necesidad de acortar los tiempos de los procesos terapéuticos está atravesada por el aumento de la demanda en los servicios de salud, las necesidades de reducir costos e incrementar la productividad en el ámbito laboral y el "estar bien" como cuestión social y moralmente reconocida. Las distintas variables señaladas convierten a estas terapias en una oferta atractiva que en los últimos años ha adquirido mayor presencia y protagonismo discutiendo con otros abordajes psi con fuerte tradición en nuestro país. La aproximación metodológica es cualitativa, se realizaron entrevistas a psicólogos/as y psiquiatras que trabajan desde el abordaje cognitivo-conductual en Buenos Aires, Argentina.

Palabras Clave: Terapia cognitivo-conductual, Tratamientos, Brevedad, Eficacia.

\section{AbSTRACT:}

Based on a research on cognitive-behavioral therapies that I am doing in the Autonomous City of Buenos Aires, the objective of this work is, from the professional's point of view, to investigate what place has brevity as an aspect that goes through the therapeutic process. From the cognitive-behavioral approach, the fact of being a psychology based on evidence; directive and focused on solving problems in the short/medium term is highlighted. So, even though treatments may be shorter or longer, there are regularities that are maintained and, in some way, pre-exist the experiences of patients. In this case, it is important to analyse the relationship between brevity and efficacy taking into account social and economic transformations in which these therapies emerge and became more popular in Argentina. First, subjects have to achieve different goals in their daily lives in a context full of difficulties and job insecurity. Secondly, because of an increase of people demand in health system there is a need to reduce time in therapeutic treatments. Taking into account these particularities, these therapies became an attractive offer that in recent years has gained a greater presence and prominence in our country. The methodological approach is qualitative; interviews were conducted with professionals working from the cognitive behavioral approach in Buenos Aires, Argentina.

KEYWORDS: Cognitive-behavioral therapy, Treatments, Brevity, Efficacy. 


\section{INTRODUCCIÓN}

Durante mucho tiempo, los cronómetros precisos y los mapas precisos han tenido el valor de su peso en oro, y el control sobre espacios y tiempos es un elemento crucial para obtener beneficios. Más aun, el dinero puede utilizarse para gobernar el tiempo (nuestro tiempo y el de otros) y el espacio. Recíprocamente, el dominio del tiempo y el espacio puede a su vez convertirse en dominio sobre el dinero (Harvey, 2017, p. 251).

El tiempo es una variable que atraviesa nuestra vida dándole forma, ritmo, duración y un sentido particular a los espacios y experiencias que habitamos. Si bien el tiempo y el espacio son categorías básicas de la existencia humana, raramente discutimos sus significados. Más bien, tendemos a darlos por sentados y a otorgarles determinaciones de sentido común (Harvey, 2017). En el campo de la salud, la temporalidad está presente constantemente, por ejemplo, al clasificar a las enfermedades en agudas o crónicas y al estipular la duración de las consultas y tratamientos.

El tiempo modela y a su vez es modelado por las experiencias de dolor y sufrimiento que pueden prolongarse o acortarse pero, más allá de eso, hay sentidos dados a la duración de los procesos en el ámbito de la salud que varían de acuerdo a la perspectiva o disciplina que se trate. Por ejemplo, los tiempos de la medicina alopática difieren considerablemente de la medicina homeopática y lo mismo sucede en el campo psi. Hay distintas concepciones sobre el tiempo que dan forma a los tratamientos terapéuticos y son atravesadas por cuestiones enmarcadas en procesos sociales, económicos, culturales y políticos propios de las sociedades contemporáneas. De esta forma, el manejo de las palabras, de los espacios, de los silencios, de la duración de las sesiones y de los tratamientos es parte y distingue a las distintas tecnologías psi entre sí.

En las psicoterapias cognitivo-conductuales, la temporalidad ocupa un lugar protagónico. Estas terapias adquieren mayor presencia en Argentina en las últimas décadas y sus desarrollos buscan diferenciarse de otras perspectivas con fuerte tradición en nuestro país como el psicoanálisis. A su vez, su surgimiento no se puede escindir o, por lo menos, no se puede analizar por fuera de un contexto de transformaciones sociales, económicas, políticas que adquieren relevancia durante la década de los noventa y que se profundizan a principios de los años 2000.

Si bien en nuestro país los inicios de las psicoterapias cognitivo-conductuales se remontan a la década del 80 (Korman, Viotti, Garay, 2010), durante varios años este abordaje ocupó un lugar marginal tanto en lo que refiere a la educación y formación universitaria como en clínicas y hospitales públicos y, recién en las últimas décadas, adquiere mayor visibilidad especialmente en Buenos Aires. Más aún, a diferencia de otros países de América latina donde el desarrollo de estas psicoterapias es significativo tanto en ámbitos públicos como privados, en Argentina su inserción ha sido más lenta y, en general hasta el momento, permanece reservada a la atención en el ámbito privado orientada a sectores socioeconómicos medios y medios altos.

En el campo psi argentino, hay estudios sobre estas terapias que historizan su desarrollo y describen las características de sus modos de diagnosticar y tratar (Keegan, 2007 y 2012; Korman, 2011; Fernández Álvarez, 2008). En cambio, desde las ciencias sociales, existen investigaciones que exploran otras tecnologías terapéuticas. A modo de ejemplo, se han encontrado estudios de relevancia que historizan y problematizan el lugar de la psicología y el psicoanálisis (Vezzetti, 1996; Galende, 1997; Visacovsky, 2002 y 2008; Dagfal, 2009; Plotkin, 2003 y 2013) y la relación entre psicoanálisis y pobreza (Epele, 2016 y 2017). Estas investigaciones permiten generar un marco conceptual de las tradiciones psi en nuestro país para problematizar el contexto en el que se inserta el abordaje cognitivo-conductual y sus interlocutores dentro de este campo.

Incluidas dentro de la categoría de terapias breves, este abordaje destaca que se trata de una perspectiva que resuelve problemas en periodos acotados de tiempo a partir de plantear objetivos concretos que intervienen y dificultan desarrollar distintas tareas en la cotidianeidad. Como se mencionó previamente, son terapias que, en nuestro país, se desarrollan especialmente en el ámbito privado. Sin embargo, el modelo de las terapias breves o de tratamientos que cuentan con una cantidad de sesiones acotadas por año está presente en distintos 
espacios del sistema de salud (especialmente obras sociales y prepagas aunque el abordaje no sea cognitivoconductual).

Desde la perspectiva de los/as profesionales, estas terapias se nombran como una "psicología basada en la evidencia" que desarrollan conocimientos siguiendo los protocolos que proponen las comunidades científicas, y que en especial tienen como modelo la biomedicina. Además, el objetivo que dicen tener es focalizar en los problemas a resolver y reducir lo más posible el tiempo de los tratamientos. Para eso, enfatizan, por ejemplo, en cifras y datos que dan cuenta de la eficacia de estas psicoterapias.

En esta relación que se establece entre conocimiento científico y eficacia, el tiempo juega un rol central porque los/as terapeutas resaltan que "lo que se investiga tiene que tener un plazo porque en algún momento tiene que terminar". Más aún, señalan: "le explicamos al paciente lo que va a involucrar el tratamiento, cuánto dura y cómo se hace".

Teniendo en cuenta lo señalado previamente, el objetivo de este trabajo es analizar el lugar que tiene la temporalidad en los relatos de los/as profesionales cognitivo-conductuales. Para eso, se explora y analiza la relación que se establece entre lo breve y eficaz como dos caras de una misma moneda, entendiendo que estos usos del tiempo no se pueden comprender independientemente de las acciones sociales y relaciones de poder implicadas en las prácticas espaciales y temporales. El aumento de la demanda en los servicios de salud por malestares psi que adquieren mayor visibilidad en las últimas décadas hace que, desde la perspectiva de los/ as terapeutas, sea necesario "contar con tratamientos que resuelven el problema en un tiempo determinado y reducen los costos”. Más aún, resaltan que los servicios de salud y gobiernos no pueden financiar aquello que "no se sabe cuándo termina", y entonces los procesos terapéuticos acotados en el tiempo se convierten en una opción recurrente. De esta forma, se indaga en las cuestiones que se esconden detrás de la relación eficacia/brevedad incorporando el contexto socioeconómico y las transformaciones de las últimas décadas como variables necesarias de tener en cuenta al momento de realizar el análisis.

A modo de síntesis, en el primer apartado se describen los inicios de las psicoterapias cognitivoconductuales a nivel mundial y, específicamente, sus desarrollos en Argentina. En el segundo apartado se analiza la relación entre brevedad y eficacia como un asunto que se convierte en sustento que legitima este abordaje psi. Al mismo tiempo, se indaga en aquellos aspectos que se dejan de lado al enfatizar la cuestión de la eficacia. Por último, en el tercer apartado, se enmarca y analiza la relación entre lo eficaz y breve teniendo en cuenta su relación con cuestiones macro que atraviesan y responden a las transformaciones sociales en las últimas décadas. El análisis de este aspecto incluye un breve señalamiento de las paradojas del caso argentino descriptas por los/as entrevistados/as al momento de mencionar los que son, hasta el momento, los campos de aplicación de estas psicoterapias en nuestro país.

\section{Metodología}

La perspectiva teórico-metodológica del estudio se inscribe en el dominio de las investigaciones desde las ciencias sociales sobre salud siguiendo los lineamientos de la investigación cualitativa. Se realizaron entrevistas a psicólogos/as y psiquiatras que trabajan desde la perspectiva cognitivo-conductual con el fin de explorar y de indagar en algunas nociones centrales de esta disciplina. En el trabajo de campo se tuvieron en cuenta algunos de los siguientes aspectos para el posterior análisis: genealogías, fundamentos teóricos, epistemológicos y modos de producción de diagnósticos, particularidades de los tratamientos (temporalidad y duración), la relación entre terapeutas/pacientes, los modelos de eficacia, concepción de sujeto que padece, entre otras cosas. En particular, para el análisis de este artículo se exploró el lugar que los/as entrevistados/ as le dan a la temporalidad y eficacia como técnicas de intervención psi y su relación con contextos macro estructurales que intervienen en los procesos de salud y atención.

Se realizaron 20 entrevistas a través de la técnica de bola de nieve a profesionales (mujeres y varones, el rango etario fue de 33-75 años) con diferentes trayectorias, experiencias y antigüedad en el campo cognitivo- 
conductual que estuvieran ejerciendo desde este abordaje en el Área Metropolitana de Buenos Aires durante el periodo octubre de 2016-abril de 2017. La decisión de acotar la población de estudio al AMBA se debe a que en dicha área geográfica se encuentra la mayor cantidad de instituciones sobre terapias denominadas cognitivo-conductuales. Las entrevistas se realizaron en consultorios e instituciones privadas (salvo en un caso en que se entrevistó a una residente de psicología en un hospital público).

Resguardos éticos: esta investigación se adecúa a los criterios de confidencialidad que se aplican en los estudios sobre salud, con el fin de asegurar los derechos de los/as participantes, así como también de resguardar su identidad. Las personas entrevistadas son mayores de 18 años. Para realizar el trabajo de campo, se llevó a cabo el proceso de evaluación requerido a través del comité de ética de una institución.

\section{INICIOS DE LAS TERAPIAS DEL “AQUÍ Y AHORA"}

Breve quiere decir que dura entre 8 y 16 sesiones durante 3 y 6 meses, más o menos, pero no vas a estar 15 años. (Psicólogo cognitivo-conductual).

La terapia cognitiva es corta, con objetivos y, si bien es un trabajo en conjunto, a diferencia de las otras terapias, se busca no generar dependencia al terapeuta. Es hacia la independencia, no hacia la dependencia de 10 años de terapia. Hacia la independencia en 6 meses, un año, dos como mucho. Lo tenés que lograr vos y seguir haciéndolo vos en realidad. De eso se trata. (Psicólogo cognitivo-conductual).

Las terapias cognitivo-conductuales surgen en Argentina a principios de la década del 80 a partir de la creación de institutos privados, grupos de discusión, congresos y jornadas. Sin embargo, recién en los años 90 y principios de los 2000 se observa una mayor presencia, institucionalización y difusión de estas terapéuticas (Korman et al., 2010). Si bien no hay estudios que analicen la relación entre el mayor protagonismo que adquieren estas psicoterapias y el contexto argentino post años 90, es importante señalar que dicho crecimiento no se puede escindir de las transformaciones que acontecieron en nuestro país en dicho periodo. Distintos estudios señalan la década del 90 como un momento de profundos cambios sociales y económicos que derivaron en una fuerte crisis a comienzo del año 2001 (Svampa, 2005; Visacovsky, 2008). Esta situación se vio acompañada de, entre otras cosas, altos niveles de desempleo y pobreza, protesta social seguida de represión estatal y descrédito de los representantes políticos (Visacovsky, 2012). Estas transformaciones repercuten en los distintos sectores sociales de manera específica modificando los modos de vida. En algunos casos, intervienen en el (no) acceso a determinados bienes y consumos mientras que en otros, a esa situación se le suman empleos temporales o, directamente, desocupación.

Esta profundización de procesos neoliberales genera incertidumbre en distintos sectores de la población y visibiliza la aparición (o mayor presencia) de una serie de malestares que son abordados desde el campo psi y se asocian con la necesidad de cumplir con distintas exigencias y demandas en situaciones de alta inestabilidad/ precariedad laboral.

Es en este contexto que el abordaje cognitivo-conductual adquiere un protagonismo que antes no tenía. Se trata de psicoterapias que resaltan el hecho de ser "breves, focalizadas y concretas" y este aspecto no es un detalle menor sino que conforma y estructura el proceso terapéutico. De hecho, desde la perspectiva de los/as profesionales, su surgimiento se da por la necesidad de buscar modelos alternativos a terapias que se prolongaban en el tiempo con el objetivo de encontrar soluciones más rápidas que respondan a las exigencias de los modos de vida actuales.

Las referencias en torno a los usos de los tiempos, las palabras, el presente y el pasado aparecen con frecuencia en las narrativas de los/as entrevistados/as y se orientan, en general, a discutir con la perspectiva psicoanalítica teniendo en cuenta su importante presencia en nuestro país. De hecho, existen numerosos estudios desde las ciencias sociales que dan cuenta de la hegemonía psicoanalítica en Argentina a lo largo del siglo XX documentando la flexibilidad que estas psicoterapias han demostrado en modelar -y ser modeladaspor diversos modos de sentir y dar sentido a experiencias subjetivas y sociales en las clases y élites medias 
y urbanas (Visacovsky, 2009; Epele, 2016). Más aún, tanto desde lo académico como desde la clínica, el psicoanálisis continua ocupando un lugar protagónico enseñándose como la perspectiva predominante en instituciones hospitalarias tanto públicas como privadas de salud (Plotkin y Visacovsky, 2007). Sin embargo, de a poco, desde lo cognitivo-conductual se comienzan a cuestionar estos saberes y perspectivas fundando instituciones y jornadas de discusión en centros urbanos como Buenos Aires con la visita de referentes cognitivo-conductuales extranjeros.

A nivel mundial, es importante tener en cuenta que este abordaje surge y se desarrolla en un momento de cambios económicos, políticos, sociales, tecnológicos. Sus inicios se remontan a la década de 1960 en Estados Unidos y se relacionan con la búsqueda de perspectivas alternativas al psicoanálisis y a las terapias comportamentales (predominantes en ese momento) para abordar malestares que estaban en aumento (y continúan hasta hoy) como la depresión y la ansiedad.

A su vez, su surgimiento no se puede escindir de una serie de transformaciones que acontecían promediando 1950 en Estados Unidos, como el desarrollo de las ciencias de la cognición, ${ }^{1}$ las analogías de la mente con las computadoras (Keegan, 2007; Korman et al., 2010) y el lugar que cada vez más comienzan a tener las ciencias basadas en la evidencia para intervenir en distintos aspectos de la vida cotidiana.

Si bien en los años 60 el puntapié inicial de estas psicoterapias fue el abordaje de estados depresivos, al poco tiempo comienzan a tratar otros padecimientos que en los últimos tiempos adquieren mayor visibilidad y no se corresponden con daños orgánicos, físicos o con las nociones tradicionales de enfermedad abordadas desde la biomedicina. En cambio, son experiencias que se asocian a estados emocionales, dificultades para conseguir determinados objetivos laborales o personales y cumplir con ciertas exigencias de los modos de vida actuales que, en general, pasan a ser diagnosticadas desde el campo cognitivo-conductual con distintos diagnósticos (trastornos de ansiedad, depresivos, fobias, trastornos obsesivos compulsivos, etc.) que se encuentran categorizados en el DSM-IV (Manual diagnóstico y estadístico de los trastornos mentales).

El aumento de estos padecimientos se estaba dando de manera exponencial y ante esta situación los servicios de salud y los gobiernos comienzan a buscar herramientas que den respuesta a las demandas de la población (no sólo por las pérdidas en los costos de salud sino también por los costos laborales de las faltas). Como señala Harvey (2017), las prácticas espaciales y temporales nunca son neutrales con respecto a las cuestiones sociales. Siempre expresan algún tipo de contenido de clase social o de luchas sociales; a modo de ejemplo, el autor señala cómo a fines de los 70 gran parte de la resistencia a los nuevos modos de trabajo había sucumbido a las presiones del desempleo y a la imposición de nuevos ritmos de trabajo. Esta aceleración de los tiempos del capital había comenzado a tener una influencia en las formas de pensar, sentir y actuar, acentuando la transitoriedad en procesos laborales, valores, etc. (Harvey, 2017, p. 257).

Siguiendo con este argumento dos terapeutas señalan:

La creación del Instituto Nacional de Salud Mental en Estados Unidos es heredero de la Segunda Guerra Mundial porque vuelven un millón de personas - más o menos- con trastornos mentales y eso genera una gran inversión del Estado en salud mental. Entonces empiezan a investigar sobre invertir en salud y el Senado pide investigación de si eso sirve o no sirve y ahí empiezan a fomentar la investigación. (Psicólogo cognitivo-conductual).

En la década de los 60,70... la segunda causa más importante de discapacidad en el mundo occidental son las enfermedades mentales. Entonces empieza a aparecer una preocupación notable por el costo social que tienen las enfermedades... la primera es la depresión... es extremadamente alta como discapacidad. Entonces un tipo se puso a pensar qué tipo de tratamiento podría ofrecer y decide diseñar un protocolo de tratamiento que en salud mental no era muy frecuente para poder medir los resultados. (Psicólogo cognitivo-conductual).

En los relatos de los/as entrevistados/as se visualiza una articulación entre el surgimiento de estas psicoterapias, los organismos de financiamiento estatales/privados y la posibilidad que tienen estas tecnologías psi de responder a las demandas de amplios sectores de la población en pos de retomar sus actividades cotidianas y "ser funcionales" ${ }^{2}$ en periodos acotados de tiempo. 
Hay dos cuestiones que se resaltan como aspectos que favorecen el reconocimiento de estas psicoterapias en distintos ámbitos y tienen que ver con atribuir a esta práctica características de cientificidad y brevedad. Más aún, dicha brevedad está sustentada empíricamente ya que hay trabajos, investigaciones, estudios que dan cuenta de la eficacia de estas psicoterapias y, este argumento, funciona para recibir financiamiento y para que en determinados países se constituyan como la opción predominante dentro del campo psi de la salud.

En este sentido, las relaciones señaladas previamente ponen de manifiesto que categorías como tiempo, conocimiento, lenguaje, entre otras, están atravesadas por procesos económicos y relaciones de poder que alteran modos de producción, relaciones laborales y vinculares. De esta forma, en el próximo apartado interesa profundizar en el análisis de la relación entre lo breve y eficaz como dos aspectos que desde esta perspectiva aparentan darse de manera conjunta y responden a criterios que siguen la ciencia basada en evidencia. En cambio, se analizan las fisuras que se esconden detrás del énfasis puesto en estas categorías como herramientas que sustentan y legitiman este abordaje psi.

\title{
EFICACIA Y BREVEDAD: DOS CARAS DE UNA MISMA MONEDA
}

\begin{abstract}
Una de las cosas que diferencia a las TCC de otros movimientos, es que busca siempre apoyo empírico. Busca apoyo empírico tanto de la eficacia de los tratamientos como de las teorías de base o si querés de la ciencia básica que de alguna manera sustenta el tratamiento. En el primer momento, la eficacia de la psicoterapia se evaluaba tomando el modelo de la medicina. (Psicóloga cognitivo-conductual).

Los modelos cognitivos son súper concretos. Es decir, todo un modelo que se adapta a un contexto y yo creo que son ideales para implementar en población hospitalaria porque no podés quedarte 50 años a perder el tiempo de la gente y porque los recursos son limitados. (Psicólogo cognitivo-conductual).
\end{abstract}

La temporalidad constituye una variable que estructura distintos ámbitos de la vida. A partir de las transformaciones en las sociedades contemporáneas asociadas con una aceleración de los ritmos, en especial en las grandes ciudades, se vincula el "no perder el tiempo" con la posibilidad de cumplir con exigencias, demandas y necesidades de la cotidianeidad en periodos acotados de tiempo. De esta forma, la posibilidad de medir y fijar la duración de actividades, cursos, encuentros y, también, terapias, aparece como algo relevante y atractivo para responder y "adaptarse”. De algún modo, lo breve aparece cada vez más vinculado con connotaciones asociadas a la capacidad de ser concretos/as, específicos/as y "no dar vueltas".

Las terapias cognitivo-conductuales captan muy bien esta necesidad desde el momento en que se presentan como terapias breves que "tienen un principio y un final". Desde estas técnicas terapéuticas se establece que hay pensamientos, comportamientos, creencias, entre otras nociones empleadas, que no permiten que el sujeto se desarrolle en distintas áreas de su vida. Entonces, uno de los objetivos es identificar dichas áreas y realizar tareas desde lo individual para alcanzar objetivos que mejoren el malestar. En algunos casos se trata de practicar pequeños hábitos o de comportarse siguiendo las pautas que dan los/as profesionales con el fin de desarrollar capacidades, competencias y modificar situaciones que generan malestar e interrumpen la cotidianeidad. Este desarrollo de competencias se plantea como cuestiones concretas que pueden ser delimitadas, medidas y resueltas en periodos cortos de tiempo. Según Rose (2012), establecer plazos más breves permite a estas psicoterapias poder auditar y evaluar los cambios realizados, ya que hay cierta satisfacción en realizar modificaciones que se puedan ver y corroborar en periodos de tiempo determinados.

De esta forma, se trata de cumplir y resolver pequeños objetivos visibles susceptibles de ser transformados en resultados, cifras, estadísticas. No es "hablar por hablar", "irse por las ramas", "hablar del pasado", sino "resolver", "pensar en el hoy", "delimitar los problemas" e "ir al punto". Estas referencias de los/as entrevistados/as intentan no sólo describir algunas particularidades de estas psicoterapias sino, también, diferenciarse de otras corrientes psi. Uno de los interlocutores "ocultos" que aparece con mayor visibilidad en las narrativas es el psicoanálisis. La búsqueda de diferenciación incluye cuestiones tan heterogéneas como 
la temporalidad, el aspecto científico, las actividades a realizar, el uso de los silencios, de las palabras, entre otras cosas.

Como se mencionó en la introducción, el surgimiento de las psicoterapias cognitivo- conductuales en Argentina fue lenta y marginal debido a la mayor presencia de tratamientos psicoanalíticos basados en la palabra y caracterizados por una descripción y análisis en detalle del hablar de los/as pacientes (Epele, 2016). Para diferenciarse de este abordaje psi, los profesionales entrevistados señalan que: "en terapia cognitivoconductual ayudamos a que esto sea más rápido entonces le vas dando escalas y el terapeuta es más activo, pregunta, cosa que en el psicoanálisis no sucede entonces te lleva años encontrar las variables a vos sola"; "no te voy a tener 20 sesiones hablando de lo mismo"; "no son terapias para toda la vida"; "otra diferencia con los psicoanalistas, nosotros le explicamos al paciente lo que va a involucrar el tratamiento, cuánto dura”.

Y yo del psicoanálisis podía entender las causas, el por qué, a lo mejor analizar toda la cuestión familiar, pero el paciente quería respuestas ya. En un paciente con pánico, ¿̨para qué me voy a meter en su infancia a ver lo que pasó? el tema es que hoy el pánico lo inhabilita para trabajar. ¿Qué me importa qué generó ese ataque de pánico si el paciente ahora no tiene vida?, si no puede ir a laburar, si no puede salir, la está pasando mal. Qué sé yo el origen, a lo mejor después lo trabajamos pero yo le tengo que mejorar su calidad de vida ahora. Entonces buscamos resultados a corto plazo, no son terapias psicoanalíticas de 20 años. (Psicóloga cognitivo-conductual).

Los plazos son más cortos en todo lo que son patologías simples. Ese es el gran problema que tuvo el psicoanálisis, hacía todo a plazo indefinible, basado en la fe y viene este tratamiento y muestra estas cosas y es un problema. Entonces como vos no investigás, no tenés fecha de finalización. Si vos querés saber cómo funciona algo, alguna vez tiene que terminar, sino no te enterás. ¿Entendés? Es como una serie que no termina nunca. No sabés qué va a pasar... si la trama sigue. Digamos, si vos querés investigar, lo primero que tiene que pasar es que en algún momento se tiene que terminar para que puedas sacar conclusión. No podés investigar durabilidad de resultados si el tratamiento no paró. (Psicólogo cognitivo-conductual).

Se ponen en juego temporalidades específicas y delimitadas del proceso terapéutico con las que buscan diferenciar y resaltar la brevedad frente a terapias prolongadas en el tiempo, el lugar "activo" de los/as terapeutas frente a un supuesto rol pasivo en otras corrientes psi, el focalizar en el presente y hablar del hoy con el objetivo de "resolver" en lugar de hablar de la historia del paciente, su infancia y eventos que supuestamente no hacen a los objetivos estipulados al comienzo de la terapia, entre otras cosas (Del Monaco, 2019a). Se trata, finalmente, de establecer una clara diferenciación entre la supuesta "fe en teorías sin sustento empírico que no se sabe cuándo terminan” frente a psicoterapias basadas en la investigación científica que se orientan a resultados en plazos determinados.

En estos relatos se comienza a vislumbrar una relación entre brevedad y eficacia como dos aspectos que se dan de la mano en las psicoterapias cognitivo-conductuales. La temporalidad está ligada a la investigación porque en la realización de estudios científicos se establecen plazos, tiempos y eso le da un marco de cientificidad al abordaje. De hecho, son constantes las referencias a que "esto es científico", "hay [x] cantidad de papers escritos al respecto, es decir, se investiga". La búsqueda de cifras y de datos que corroboren esta disciplina se enmarca dentro de un contexto en el cual los números y las probabilidades se convierten en una fuente de legitimidad y credibilidad (Del Monaco, 2019a). Siguiendo a Hacking (2011), las probabilidades y las estadísticas permean diferentes ámbitos de la cotidianeidad y detrás de este fenómeno se encuentran las nuevas técnicas de clasificar, enumerar y categorizar.

En el caso de estas psicoterapias, el lugar que tienen como marcos de referencia disciplinas como la biomedicina y ciencias naturales es central y se traduce, también, en los modos de nombrar y de intervenir terapéuticamente. Apelar a la evidencia y a teorías comprobadas empíricamente como herramientas durante los tratamientos hace que estos lenguajes comiencen a permear, también, en distintos ámbitos del sentido común de capas de racionalidad y objetividad, edificando un tipo de saber que goza de una serie de privilegios asociados con la objetividad y neutralidad científica. Esta búsqueda de sustentar la práctica psi en saberes basados en la evidencia muestra que los modelos de saber y verdad hegemónicos en las sociedades occidentales contemporáneas están mediados por un tipo de conocimiento científico que busca universalizar y generalizar resultados. 
En este sentido, una categoría recurrente en los relatos de los/as entrevistados/as para describir a estas psicoterapias es la noción de eficacia. De acuerdo con la RAE, la eficacia es la capacidad de lograr el efecto que se desea o se espera. En el caso de los/as terapeutas cognitivo-conductuales la eficacia proviene de una serie de investigaciones previas que dicen corroborar no sólo que se han realizado estudios cuyos resultados muestran que son terapias "que funcionan", sino que también se establece que hay temporalidades específicas en torno a dicha resolución. Esta cuestión reviste a esta práctica, de manera casi inmediata, de una aparente legitimidad y seguridad en torno a sus resultados que los/as entrevistados/as resaltan como un aspecto que los/as diferencia de otras tecnologías psi.

A partir del 95 empiezan los estudios y se acomodaron tal cual a lo que eran las normas generales de los estudios basados en la evidencia que provenían de la medicina y es un tema de debate porque ese es un modelo que camina sobre dos patas, digamos, ¿no?, para determinar la eficiencia de un tratamiento. Si uno quiere estudiar cuál es la eficiencia que tiene una intervención terapéutica, hay dos métodos para hacerlo: una es la eficacia y la otra es la efectividad. La eficacia funciona con muestras controladas y aleatorias, mientras que la efectividad funciona con muestras naturales. Entonces al funcionar la eficacia con muestras controladas le da una validez interna muy grande al procedimiento, y en medicina eso es lo que más predomina. Ahora, cuando lo trasladas al caso de la psicoterapia, es muy potente lo que informa, pero tiene poca validez externa, porque en realidad... son esos famosos grupos de muestra que se arman con personas que terminan siendo, como decimos nosotros en la jerga interna, estudiantes universitarios, que son los que están disponibles. Es decir, son poco reales, pero son buenas muestras, están todas bien pensadas, están todas depuradas, se pueden hacer estudios aleatorizados... comparando una muestra de estudio, con una muestra control, todo perfecto, pero, no son los pacientes que vemos. Entonces hoy por hoy hay una gran discusión y yo formo parte de un grupo que estamos peleando contra la tiranía de la eficacia, lo que pasa que los organismos, que financian todo esto, tienden a apoyar siempre los estudios de eficacia, porque son los típicos estudios médicos. (Psicólogo cognitivo-conductual).

No obstante, lo que muestran las palabras del profesional es que las constantes referencias en torno a la eficacia como elemento que justifica estos tratamientos por sobre otros, en realidad, se encuentran determinadas por una serie de variables que no siempre responden ni incluyen a los distintos sectores sociales, contextos y poblaciones a las que se aplican estos criterios de eficacia. De hecho, una de las cuestiones que se resaltan en este relato, y que también aparecen en las narrativas de otros/as profesionales, refiere a los modos en que se conforman las muestras y a la dificultad para realizar estudios con sujetos heterogéneos entre sí. Es decir, al hablar de eficacia se hace referencia a la posibilidad de obtener resultados que son empleados para destacar las bondades de estas psicoterapias, pero estos datos en gran parte están alejados de las realidades de poblaciones diferentes entre sí. Más aún, lo que deja por fuera la búsqueda de imitar el modelo biomédico basado en la investigación científica son las múltiples experiencias de quienes padecen.

Entonces, establecer una relación cuasi lineal entre la eficacia y brevedad como sustento y aspectos que caracterizan a este abordaje psi pone de manifiesto que las referencias a lo eficaz, en algunos casos, pueden funcionar como argumentos para justificar la brevedad de los tratamientos. Siguiendo este esquema, más allá del "problema" a resolver, el tratamiento tiene una duración que se puede acortar o prolongar, pero que bajo ningún punto de vista excederá determinados plazos. En estos casos, como se analiza en mayor profundidad en el próximo apartado, lo que se deja de lado y se esconde detrás de la noción de eficacia es que la brevedad está atravesada y modelada por cuestiones macroestructurales que responden a las demandas de los servicios de salud, de los gobiernos y de las empresas privadas de salud en torno a los tiempos de los tratamientos que se financian.

\section{FUNCIONALIDAD Y ADAPTACIÓN (DE LOS TRATAMIENTOS AL CONTEXTO)}

En la actualidad, algunas investigaciones analizan cómo la psicología, en especial aquella que se desarrolla en hospitales y en centros de salud, es afectada por cuestiones socioeconómicas que demandan de investigaciones que den cuenta de los costos, beneficios y eficacia de los tratamientos. En este sentido, un análisis comparativo entre la cantidad de estudios publicados en Chile y Argentina muestra que, si bien dicha producción es escasa, 
las terapias cognitivo-conductuales son las que más investigan y producen conocimiento científico que aporta datos para pensar en términos de intervención (Villarroel y Mustaca, 2006). Estudios en Brasil, también, focalizan en la importancia dentro de los servicios públicos de salud de adaptar los tratamientos tradicionales a las modificaciones del contexto y, para eso, resaltan el lugar de las terapias cognitivo-conductuales como intervenciones que "respetan" las posibilidades de los contextos públicos como la falta de recursos, los números elevados de la demanda, el tiempo de las sesiones, etc. Más aún, en estos trabajos señalan a la brevedad y objetividad como cualidades y herramientas valiosas que contribuyen a las intervenciones de los/ as terapeutas en contextos en los que se conjuga la alta demanda con los tiempos de atención reducidos (De Souza Silva, Cavalcanti Pereira, Avellar de Aquino, 2011, p. 44-49).

Han tenido la virtud o la ventaja, depende de cómo uno lo quiera mirar, de construirse como propuestas terapéuticas que fueron muy porosas, facilitaron enormemente la investigación empírica y le permitieron encajarse, digamos, en el tronco de las prácticas basadas en la evidencia. Entonces el $90 \%$ de los estudios empíricos, el $90 \%$ de las investigaciones que han reunido pruebas de evidencia en el campo de la psicoterapia, son de origen cognitivo conductual. Entonces eso, claro, a nivel de los órganos de gobierno y de los órganos que regulan los fondos en salud, siempre es lo que recibe los fondos y subvenciones...van primero ahí porque hay un método que lo hace fácil, porque las terapias se pueden plantear de una manera muy formalizada, se pueden plantear de una manera muy acotada, muy manualizada, entonces así es más fácil hacer estudios sobre eficacia. (Psicólogo cognitivo-conductual).

Vos vas a cualquier municipio de Inglaterra y tienen estos tratamientos gratis y el Estado los controla online sesión por sesión, cada centro tiene que tener una tasa de recuperación del 45 \% y si bajan de eso, les mandan a un tipo que los ayuda para subir. El estudio económico hecho y el impacto del tratamiento demuestran que es mucho más barato el tratamiento que los gastos que esa persona tendría para con la comunidad en caso de continuar con la patología. (Psicólogo cognitivoconductual).

Las nociones de funcionalidad y adaptación aparecen con frecuencia en los relatos de los/as profesionales cognitivo-conductuales. Como se mencionó en el primer apartado de este trabajo, estas nociones se suelen aplicar desde este abordaje psi para pensar la relación de los sujetos con el "medio ambiente". Resulta interesante retomar estas ideas para abordar y problematizar la relación de estas psicoterapias con los distintos contextos en los que surgen y se desarrollan. El énfasis puesto en que "estas terapias resuelven problemas reales" está vinculado a un modo de concebir malestares; un modo que pone el foco en cuestiones concretas que intervienen en la cotidianeidad y que se pueden delimitar y abordar en un plazo de tiempo determinado. Por eso, apelar a que se trata de "terapias activas" está asociado a la importancia de "hacer frente a miedos/ temores/problemas", ya que el acento está puesto en resolver para "adaptarse y ser funcional".

Si bien desde este campo psi se habla de la importancia de responder a las problemáticas de los sujetos en determinados tiempos, este asunto se enmarca en condiciones macro que incluyen distintos actores e instituciones. Algunos de los elementos que se ponen en juego refieren a las exigencias de los servicios de salud para responder a las demandas de la población en periodos breves de tiempo, a ser psicoterapias atractivas para los organismos de financiamiento, a disminuir los costos por enfermedad en los lugares de trabajo e incrementar la productividad, y a que los sujetos estén "bien y saludables" en el menor tiempo posible. Es un contexto donde la búsqueda de eficiencia en el sistema productivo se centra en reducir los tiempos y en incrementar lo más posible la adecuación y flexibilidad de los/as trabajadores/as (quienes experimentan periodos de empleo/desempleo/reestructuración, etc.) para responder a las exigencias de la competitividad y en los ámbitos laborales (Harvey, 2017).

Si vos tenés alguna evidencia que funciona, sale una medicación para el HIV y en 24 horas el mundo entero está recibiendo eso. Pero sale una nueva intervención en psicoterapia y tarda 20 años en bajar al mundo real. Es así. Si yo pago 100 sesiones para curar y me dicen: "No, mirá que con 10 está todo bien" y yo soy del gobierno, ¿a quién voy a contratar?, a los que me ofrecen las 10 sesiones con los mismos resultados, al más rápido. (Psicólogo cognitivo-conductual).

La investigación de psicoterapia en el mundo desde la década de los 80 hasta ahora es quizás una de las áreas de desarrollo de investigación que más ha crecido en la historia, básicamente por la demanda social de tratamientos basados en la evidencia y porque los gobiernos empiezan a pedir resultados. Especialmente gobiernos que tienen sistemas públicos de salud como Inglaterra, empieza a decir: "yo lo voy a pagar, pero quiero ver si funciona”. (Psicólogo cognitivo-conductual). 
Desde la perspectiva de los/as entrevistados/as se articulan distintas cuestiones aparentemente alejadas entre sí que se pierden de vista cuando se concibe o se intenta reducir la modalidad de tratamiento breve a la relación profesional-paciente. Expresiones como gobierno, financiamiento, tratamientos basados en la evidencia, pedir resultados, estudios económicos, tasa de recuperación, investigación, entre otras, visibilizan un tipo de abordaje que intenta responder a demandas a nivel macro. De hecho, como se mencionó en el primer apartado, estas psicoterapias surgen, por ejemplo, en Estados Unidos, a partir de un incremento significativo de la población con malestares asociados a estados depresivos que necesitaban ser abordados desde el campo psi con tratamientos que den respuestas "rápidas" para que los sujetos puedan volver a sus actividades habituales lo antes posible.

A partir de las entrevistas realizadas y de otras fuentes secundarias como investigaciones desde el campo psi, se pone de manifiesto que estas psicoterapias modelan un tipo de tratamiento que busca ser funcional a las exigencias de las sociedades actuales. Lo particular es que el sustento de la brevedad está dado por las referencias empíricas en torno a la eficacia. No obstante, esta articulación entre eficacia y brevedad deja de lado u oculta que los gobiernos y los servicios de salud no pueden financiar la indeterminación y, por eso, en distintas partes del mundo y cada vez más en América Latina estos tratamientos se convierten en herramientas atractivas que hablan de tener un "inicio y final donde se resuelve el problema".

Entonces, son el contexto social, económico, los recursos disponibles, la búsqueda de maximizar ganancias, reducir costos y de responder a las demandas de la población algunos de los aspectos que modelan y dan forma a los tratamientos breves o de corto plazo. El tema es que todos estos aspectos quedan parcialmente invisibilizados y ocultos en la noción de eficacia. Esta categoría permite justificar duraciones y plazos teniendo en cuenta que el desarrollo en un tiempo determinado está avalado y justificado empíricamente. Siguiendo a Rose (2012), estos "regímenes de verdad" que se esconden detrás de estos lenguajes garantizan determinadas prácticas, procedimientos y modos de intervención. Por eso, es necesario explorar y cuestionar los sentidos únicos dados al tiempo visibilizando el lugar de las prácticas, las transformaciones sociales, económicas, políticas en los modos de definirlo y de abordarlo para la reproducción de la vida social. Es decir, las referencias constantes a que se está en presencia de "terapias breves que resuelven problemas de manera eficaz y que esa eficacia está sustentada en estudios basados en el método científico", lo que omite son las múltiples variables que intervienen en la definición de los tiempos de los tratamientos. Más aún, y retomando uno de los relatos de los/as entrevistados/as, la "tiranía de la eficacia” lo que hace es dar resultados que no siempre se corresponden con las situaciones en los contextos particulares de cada proceso terapéutico.

\section{Las paradojas del caso argentino...}

El sistema de salud de la Argentina es un desastre, no tiene ninguna relación con las necesidades de la gente y cambiarlo es muy complicado porque la inmensa mayoría de los psicólogos tienen un fervor lacaniano. A pesar de que eso ahora está cambiando un poco los terapeutas cognitivos conductuales no duran en las obras sociales porque tienen mucho trabajo en el ámbito privado. (Psicólogo cognitivo-conductual).

En las mejores obras sociales hay, lo que pasa es que te diría que los que son muy buenos pueden seguir trabajando en lo privado. Esto todavía no llegó a ser como Favaloro con el bypass que antes no te querías operar con cualquiera y hoy llegas a cualquier lado y te dicen "hay que hacer un bypass" y lo hacés. Todavía estamos lejos de que sean terapias más masivas. (Psicóloga cognitivo-conductual).

Si bien en este trabajo se indagó sobre el lugar que los/as profesionales le dan a las nociones de brevedad y eficacia como aspectos centrales de este abordaje psi, interesa señalar brevemente algunas cuestiones que se diferencian y destacan -según sus narrativas- en el sistema de salud argentino. No es objetivo de este escrito realizar un análisis exhaustivo de este aspecto ya que el mismo requiere de un desarrollo aparte a futuro. Sin embargo, la búsqueda de convertir a estas psicoterapias en tecnologías que respondan a las demandas de la población de manera más global y estructurada no se corresponde con las características del sistema de salud en nuestro país. Los/as entrevistados/as resaltan, entre otras cosas, que "si vos hacés una estadística en Buenos Aires, la terapia cognitiva sigue siendo infinitamente menor...", "cuesta más que haya profesionales que hacen 
TCC en las prepagas y obras sociales" y, que "el Estado tiene una cuota de responsabilidad porque no da difusión entre la población sobre que existen tratamientos específicos para determinadas condiciones".

\begin{abstract}
Cuando nosotros empezamos en el campo psi teníamos una posición absolutamente marginal, nos tiraban con tomates. Con el paso del tiempo dejamos de estar en un lugar marginal y empezamos a estar en la periferia. Hace unos 20, 25 años -creo yo- que estamos en el centro, ahora somos respetados, pero eso y que haya igualdad de condiciones para la difusión, no. Porque todavía hay un predominio del psicoanálisis, o sea, vas a centros de salud mental y trabajan no sé 500 personas y 490 son psicoanalistas. Bueno, pero eso va a llevar su tiempo. Finalmente va a ocurrir porque hay cosas que pasan en la Argentina que son situaciones excepcionales con respecto al resto del mundo. No todos pueden ir a una práctica privada, entonces tendrían que tener chances de que en un hospital o centro de salud tuvieran tantos terapeutas cognitivos como psicoanalistas. (Psicólogo cognitivo-conductual).
\end{abstract}

A diferencia de otros países en los que las psicoterapias cognitivo-conductuales aparecen como técnicas psi desde los organismos públicos y privados de salud para dar respuestas "rápidas" a poblaciones en las que aumentan (o se hacen más visibles) malestares que dificultan o impiden continuar con las actividades cotidianas, en Argentina esta situación todavía no alcanza esa difusión y presencia. De hecho, las referencias que dan los/as entrevistados/as son a nivel mundial, como Estados Unidos e Inglaterra, y a nivel local, en América Latina, países como Chile o Brasil donde la presencia de estas psicoterapias ha ido en aumento en las últimas décadas tanto en la formación como en la clínica.

Sin embargo, lo particular en nuestro país es que si bien las psicoterapias cognitivo-conductuales no aparecen como una de las terapias de mayor presencia en los distintos ámbitos de salud, sí existe una característica que se replica de este modelo, en especial en obras sociales y prepagas, que se relaciona con la cantidad de sesiones cubiertas por año y con la búsqueda de trabajar "por objetivos". El esquema de la terapia breve y orientada a objetivos permea distintos ámbitos de la salud y responde a un intento de reducir costos y financiar tratamientos que puedan concluir en periodos acotados de tiempo. Tanto en las principales prepagas como en algunos hospitales privados ubicados en Ciudad de Buenos Aires, la cantidad de sesiones que son parcialmente cubiertas (ya que se abona un copago ${ }^{3}$ ) son 30 por año. Luego, en caso de que el/la paciente continúe debe abonar la totalidad de la consulta. En cambio, en los servicios públicos de salud es más complejo definir una cantidad de sesiones determinada durante el tratamiento. Profesionales del campo psi señalan que "en los servicios públicos los tratamientos que se ofrecen son más a pacientes con diagnósticos puntuales y, además, hay más una primacía de la emergencia porque hay mucha demanda y poco personal”.

En nuestro país se trata de psicoterapias a las que pueden acceder personas de sectores socioeconómicos medios y medios altos ya que, en general, se realizan en consultorios privados. Entonces, ante el incremento de la competitividad, precariedad y procesos de inclusión/exclusión en distintos ámbitos de la vida cotidiana, la posibilidad de responder "rápidamente" a las demandas del sistema continúa siendo una cuestión que atraviesa a distintos sectores, siendo sólo algunos pocos quienes pueden responder a dichas demandas en los plazos exigidos. De esta forma, alcanzar cierto "bienestar" y "funcionalidad con el medio" se convierte y reduce a una búsqueda por resolver problemas de la manera más rápida y eficaz posible.

\title{
Conclusiones
}

Siempre son terapias muy focalizadas en el presente, en el aquí y ahora porque lo que te pasa te pasa hoy. (Psicóloga cognitivoconductual).

Entonces cuando hablamos de basados en la evidencia, en depresiones cuántos días menos de cama, cuántos días más el paciente trabaja, cómo son sus relaciones de intimidad. Son siempre variables cuantitativas. Es decir, yo puedo en una intervención psicosocial medir cuántos días pasa en la cama. (Psicólogo cognitivo-conductual).

A partir de una serie de transformaciones en las últimas décadas vinculadas a cambios en los tiempos laborales, en las formas de vincularnos, en los modos en los que circula la información e imágenes, entre otras cosas, la instantaneidad adquiere connotaciones positivas asociadas al "focalizar en el hoy". En este sentido, 
al mismo tiempo que la posibilidad de transmitir un mensaje de la manera más concreta y breve posible se convierte en un valor celebrado como la posibilidad de "síntesis" y de "ir al punto", aquellos casos donde los plazos no están definidos, donde se habla del "pasado en lugar del focalizar en el presente” y "se dan vueltas", se revisten de connotaciones negativas.

Desde el campo psi, las terapias cognitivo-conductuales surgen y adquieren mayor protagonismo en estos contextos y, de hecho, uno de sus presupuestos es "dar respuestas más rápidas a procesos de dolor y sufrimiento que alteran la cotidianeidad, no son funcionales ni permiten una adaptación al medio". Las exigencias de "estar bien" lo antes posible para retomar las actividades habituales aparecen como una cuestión recurrente de los/as entrevistados/as al momento de asociar estas psicoterapias con los modos de vida de las sociedades neoliberales. De hecho, como se menciona a lo largo del escrito, el auge de estas psicoterapias en nuestro país se enmarca en un contexto de profundas modificaciones sociales, culturales y económicas post años 90. Como señala un entrevistado: "estamos en una sociedad acelerada, de consumo, que necesita estos modelos". Entonces, se presentan como terapias "manualizadas" que, siguiendo una serie de pasos, tareas, presupuestos dados por los/as terapeutas, permiten resolver malestares que interfieren en la cotidianeidad en plazos relativamente cortos de tiempo. El aspecto que sobresale y que se analizó en este trabajo tiene que ver con que el énfasis puesto en la brevedad aparece, en los relatos de los/as terapeutas entrevistados/as, atado a la cuestión de la eficacia. Es decir, hay saberes legitimados que sustentan el modo de investigar y dar respuestas en las intervenciones psi que no se cuestionan y que tienen que ver con una tradición de estudio donde conocimiento y verdad aparecen, también, como dos caras de una misma moneda. Sin embargo, explorar la relación entre lo breve y eficaz permitió no sólo cuestionar la eficacia como una noción que al utilizarse como argumento puede descontextualizar y objetivar los problemas por los que se consulta sino que, al mismo tiempo, en esta lógica se establecen plazos que preexisten a las experiencias de quienes padecen.

En este sentido, más allá de las investigaciones realizadas y los resultados que arrojan las probabilidades en torno a la eficacia de estas psicoterapias en determinados malestares, se invisibilizan los modos en que las condiciones estructurales modelan e intervienen en los tratamientos psi, específicamente en las formas que adquieren de acuerdo a los contextos respondiendo a demandas que exceden ampliamente a quienes consultan. Es decir, al articular la eficacia con la brevedad como argumento para legitimar estas técnicas psi en los sistemas de salud, porque "resuelven problemas en periodos acotados de tiempo", lo que se termina invisibilizando es que el sustento en datos, cifras y probabilidades busca justificar la brevedad y rapidez de los tratamientos. En cambio, interesa plantear como el tiempo nos atraviesa e interviene en los distintos espacios que habitamos de distintas maneras y no se puede analizar y concebir como una cuestión aislada de los sistemas sociales, de los modos de producción, de las relaciones con los organismos de salud, de financiamiento, de gobierno.

En el caso de las tecnologías psi, la temporalidad es una variable central que, dependiendo del abordaje terapéutico, ocupa distintos roles. En las psicoterapias cognitivo conductuales, el énfasis en la brevedad se convierte en una herramienta de intervención que estructura los tratamientos ya que hay plazos, inicios, finalizaciones que hay que cumplir y, todo eso, está basado en presupuestos que siguen investigaciones científicas. Entonces, el énfasis puesto en el binomio eficacia-brevedad oculta otros argumentos por los que es necesario acortar los tratamientos y, a través de los cuales, también, se obturan y acortan los procesos y experiencias que intervienen en la producción de malestares y sufrimientos.

\section{ReFERENCIAS}

Dagfal, A. (2009). Entre París y Buenos Aires. La invención del psicólogo (1942-1966). Buenos Aires: Paidós.

Del Monaco, R. (2019a). Colorear racionalmente el mundo. Nociones de creencia implicadas en las terapias cognitivas conductuales en Buenos Aires, Argentina. Aposta. Revista de Ciencias Sociales, 81, 137-150. 
Del Monaco, R. (2019b). Ir al punto, definir plazos y objetivos: tiempos y palabras en los tratamientos cognitivoconductuales en Buenos Aires, Argentina. Runa, 40(1), 65-82.

De Souza Silva, S., Cavalcanti Pereira, R., Avellar de Aquino, T. (2011). A terapia cognitivo-comportamental no ambulatório público: possibilidades e desafios. Revista Brasileira de terapias cognitivas, 7(1), 44-49.

Epele, M. (2016). El hablar y la Palabra Psicoterapias en los Márgenes de Buenos Aires. Revista Antípodas, 25, 15-31.

Epele, M. (2017). Sobre las Posiciones Etnográficas en la Antropología de la Salud en el Sur de las Américas. Revista salud colectiva, 13(3) 359-373.

Fernández Álvarez, H. (2008). Integración y salud mental. España: Desclée de Brouwer.

Galende, E. (1997). De Un Horizonte Incierto. Psicoanálisis y Salud Mental en la Sociedad. Buenos Aires: Paidós.

Gardner, H. (2011). La nueva ciencia de la mente. Historia de la revolución cognitiva. México: Paidós.

Hacking, I. (2011). La domesticación del azar. La erosión del determinismo y el nacimiento de las ciencias del caos. Barcelona: Gedisa.

Harvey, D. (2017). La condición de la posmodernidad: investigación sobre los orígenes del cambio cultural. Buenos Aires: Amorrortu.

Keegan, E. (2007). Ensayos de Terapia Cognitiva. Buenos Aires: EUDEBA.

Keegan, E. (2012). La salud mental en la perspectiva cognitiva conductual. Vertex. Revista Argentina de Psiquiatría, 23(101), 52-56.

Korman, G., Viotti, N., Garay, C. (2010). Orígenes y profesionalización de la terapia cognitiva. Algunas reflexiones sobre la configuración del campo psi en Buenos Aires. Revista Argentina de Clínica Psicológica, 19(2), 141-150.

Korman, G. (2011). Bases teóricas en la conformación de la terapia cognitiva en Argentina. Revista Interamericana de Psicologia/Interamerican Journal of Psychology, 45(2), 115-122.

Pinker, S. (2001). Cómo funciona la mente. Barcelona: Ediciones Destino.

Plotkin, M. (2003). Freud en las Pampas. Buenos Aires: Editorial Sudamericana.

Plotkin, M. y Visacovsky, S. (2007). Saber y Autoridad en las intervenciones de los psicoanalistas en torno a la crisis en la Argentina. EIAL, 18(1), 11-27.

Plotkin, M. (2013). Historia y Psicoanálisis. Encuentros y desencuentros. CulturasPsi/PsyCultures, 1(2), 25-44.

Rose, N. y Miller, P. (2008). Governing the present. Administering economic, social and personal life. USA: Polity Press.

Rose, N. (2012). Politicas de la vida: biomedicina, poder y subjetividad en el siglo XXI. La Plata: UNIPE: Editorial Universitaria.

Svampa, M. (2005). La Sociedad Excluyente. La Argentina bajo el signo del Neoliberalismo. Buenos Aires: Taurus.

Thagard, P. (2010) La mente. Introducción a las ciencias cognitivas. Madrid: Katz Editores.

Vera Villarroel, P. y Mustaca, A. (2006). Investigaciones en psicología clínica basadas en la evidencia en Chile y Argentina. Revista Latinoamericana de psicologia, 38(3), 551-565.

Vezzetti, H. (1996). Freud en Buenos Aires. Universidad Nacional de Quilmes.

Visacovsky, S. (2002). El Lanús. Memoria y Política en la Construcción de una Tradición Psiquiátrica y psicoanalítica argentina. Buenos Aires: Alianza Editorial.

Visacovsky, S. (2008). Estudios sobre "clase media" en la antropología social: una agenda para la Argentina. Avá Revista de Antropología, (13), 9-37.

Visacovsky, S. (2009). La constitución de un sentido práctico del malestar cotidiano y el lugar del psicoanálisis en la Argentina. Cuicuilco: Revista de la Escuela Nacional de Antropología e Historia, 16(45), 51-78.

Visacovsky, S. (2012). Experiencias de descenso social, percepción de fronteras sociales e identidad de clase media en la Argentina post-crisis. Pensamiento Iberoamericano, (10), 133-168. 
Romina Del Monaco. "Terapias del aQuí y ahora porque lo que te pasa te pasa hoy": brevedad y EFIC...

\section{Notas}

1 Los desarrollos de las ciencias de la cognición a partir del estudio y búsqueda de explicaciones sobre el funcionamiento de nuestra mente se constituyeron en un campo teórico amplio que abarca distintas áreas de investigación tales como la antropología, psicología y lingüística (Pinker, 2012; Thagard, 2010). En sus inicios, planteaban un tipo de sujeto universal teniendo como objetivo decodificar las características de sus conductas a partir de conocer los esquemas de pensamiento que intervenían en ellas (restando importancia a ciertos hechos como la influencia de distintos contextos socioeconómicos) (Gardner, 2011).

2 De acuerdo a los relatos de los/as psicólogos/as y psiquiatras entrevistados/as, la noción de funcionalidad refiere a "la posibilidad de ejecutar una acción para la que se está preparado" (Del Monaco, 2019b).

3 Copago es un concepto que se utiliza en el ámbito de la medicina privada para definir la diferencia que existe entre el precio de un servicio y el valor del mismo que cubre un plan de salud. Se trata, por lo tanto, del monto que tiene que pagar el afiliado para acceder a dicho servicio.

\section{BY-NC-SA}

